\title{
Exploring Electronic Medical Record and Self-Administered Medication Risk Screening Tools in a Primary Care Clinic
}

\author{
Mark J. Makowsky, BSP, PharmD; Ken Cor, PhD; and Tat Wong, BScPharm
}

\begin{abstract}
BACKGROUND: Electronic medical record (EMR) screening for indicators of medication risk could improve efficiency in identifying primary care clinic patients in need of clinical pharmacist care compared with patient selfreporting.
\end{abstract}

OBJECTIVES: To (a) compare the performance of an EMR medication risk assessment questionnaire (MRAQ) with a self-administered (SA) MRAQ and (b) explore each tool's ability to predict indicators of health behavior, health status, and health care utilization.

METHODS: A prospective cohort study was conducted with 143 adults who attended an academic family medicine center and were taking $\geq 2$ medications. All participants completed the 10-item SA-MRAQ, Morisky Medication Adherence Scale, Chew's health literacy screener, Stanford Health Distress Scale, and SF-36 overall rating of health. A blinded investigator completed the EMR-MRAQ and a chart review to ascertain 6 months of health care utilization. Outcome measures included the following: (a) scores from the 5- and 10-item SA-MRAQs and 5-item EMR-MRAQ; (b) sensitivity and specificity to determine the accuracy of the 5-item EMR versus the 5-item SA risk scores; (c) correlations between risk assessments and health behavior/status scales; and (d) area under the receiver operator curve to determine how well a high-risk score predicted health care utilization.

RESULTS: The 5-item SA-MRAQ, the 5-item EMR-MRAQ, and the 10-item SA-MRAQ categorized $52.9 \%(55 / 104), 69.2 \%$ (99/143), and $17.6 \%$ (18/102) of participants as high risk, respectively. For the 104 participants who completed both 5-item MRAQ tools, the EMR-MRAQ had a sensitivity of $81.8 \%$ and specificity of $49.0 \%$ in detecting a high-risk SA-MRAQ score. Both 5-item risk assessments showed weak correlations with health distress and overall health, while the 10-item SA-MRAQ additionally showed weak correlations with medication adherence. The EMR-MRAQ was most effective in predicting all-cause emergency room visits/hospitalization (c-statistic $=0.69 ; 95 \% \mathrm{Cl}=0.57-0.81$ ) and high clinic utilization ( $\geq 4$ visits per 6 months; c-statistic $=0.77 ; 95 \% \mathrm{Cl}=0.69-0.85$ ). The EMR-MRAQ had high sensitivities but low specificities for these health care utilization outcomes, respectively (82.6\% and $33.3 \% ; 88.9 \%$ and $42.7 \%$ ).

CONCLUSIONS: This pilot study suggests that EMR-MRAQ screening has high sensitivity but low specificity in comparison with self-reporting and was able to discriminate between those who would and would not experience health care utilization outcomes. These results justify further development and validation of an automated EMR-based tool to predict patientimportant consequences of medication-related problems.

J Manag Care Spec Pharm. 2017;23(5):566-72

Copyright $\odot 2017$, Academy of Managed Care Pharmacy. All rights reserved.

\section{What is already known about this subject}

Adverse drug events account for a large proportion of preventable emergency room visits and hospitalizations.

Having patients complete a validated screening tool for medication-related problems during primary care clinic visits can be helpful in prioritizing patients for pharmacist consultation in medical practices but can be challenging to implement in practice. An automated clinical decision support system embedded in an electronic medical record (EMR) could potentially allow for proactive and highly efficient screening of a large panel of primary care clinic patients, alerting clinicians to high-risk patients and triggering referral to a pharmacist, which ultimately could result in improved patient outcomes.

\section{What this study adds}

This study explored the feasibility of using a primary care clinic EMR review to estimate medication risk by investigating performance of EMR and self-administered risk assessment questionnaires on indicators of health behavior, health status, and health care utilization.

Results suggest that EMR-based risk assessment had high sensitivity and low specificity in detecting patients classified at high risk by self-reporting, weakly correlated with health distress and overall health, and was effective in detecting the composite of at least 1 emergency room/hospital visit in 6 months and high clinic utilization, with high sensitivity but low specificity.

Further development and validation of a primary care clinic EMR-based tool to predict patient-important consequences of medication-related problems is warranted.

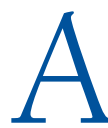
dverse drug events (ADE) are common in ambulatory care and account for a large proportion of preventable emergency room (ER) visits and hospitalizations. ${ }^{1-7}$ Between $2.8 \%$ and $34.7 \%$ of ambulatory care patients experience 1 or more ADEs, of which $16.5 \%$ are potentially preventable. $^{3}$ Additionally, an estimated $5 \%-25 \%$ of all hospital admissions, and up to $12 \%$ of ER visits, are medication related, of which $50 \%-70 \%$ are preventable., ${ }^{4,5}$ Pharmacists providing direct patient care in primary care medical practices, such as those modeled on the patient-centered medical home, represent a strategy to reduce preventable drug-related morbidity 
because of ADEs. ${ }^{8,9}$ However, high patient-to-pharmacist ratios make it important to identify which patients will benefit most from consultation with a primary care pharmacist in order to best use pharmacist resources.

Several screening tools have been developed to identify ambulatory patients at risk of experiencing medication-related problems across care settings, ${ }^{10-23}$ and most tools are patient self-administered. ${ }^{15-23}$ Criteria from these tools have been used to establish eligibility for medication therapy management services in the United States and similar services in Canada. ${ }^{24,25}$ We previously identified issues in using patient self-administered risk screening tools and recognized that increased uptake of electronic medical records (EMRs) in family physician offices could potentially allow for proactive, automated, and highly efficient screening of a large number of patient records for medication-related risk. ${ }^{21}$ An automated clinical decision support system (CDSS) to detect medication risk in a primary care clinic could alert clinicians to high-risk patients and trigger referrals to a pharmacist or other interdisciplinary team members, which ultimately could result in improved patient outcomes.

As a first step towards development of a medication-risk CDSS, the objectives of this pilot study were to (a) assess the accuracy of an EMR-based medication risk assessment questionnaire (MRAQ) in comparison with a self-administered (SA) MRAQ and (b) explore each tool's ability to predict indicators of health behavior, health status, and health care utilization.

\section{Methods}

\section{Setting and Patients}

This prospective cohort study was carried out at the Royal Alexandra Family Medicine Centre, a university-affiliated academic teaching clinic located in Edmonton, Alberta, Canada. The study occurred on selected full and half clinic days between February 24, 2014, and April 16, 2014. Clinic patients who were aged $>18$ years, were taking $\geq 2$ medications, and spoke English were eligible for inclusion. Patients were excluded if they were unable to complete the survey on their own (e.g., patient-reported dementia, illiteracy, or emergent medical problems) or were not responsible for management of their own medications.

\section{Medication Risk Assessment Questionnaire}

We administered a revised version of our 10-item MRAQ to patients as the SA-MRAQ (Table 1). ${ }^{21}$ All questions were included, but dichotomous response options were converted into continuous scales to explore the tool's discriminatory power. After reviewing previous literature and evaluating each of the SA-MRAQ criteria for ease of abstraction, we included only 5 items, resulting in the creation of the 5-item EMRMRAQ and its equivalent 5-item SA-MRAQ. ${ }^{19,20,23}$

\section{Study Protocol}

A research assistant solicited informed consent from consecutive patients attending the clinic. Patients completed the full SA-MRAQ, the 8-item Morisky Medication Adherence Scale (MMAS-8), 3 health literacy screening questions, the Stanford Health Distress Scale, the single overall rating of health in the past 4 weeks from the SF-36, and provided demographic information including age, gender, ethnicity, employment status, education level, and annual household income. ${ }^{26-28}$ Those who returned a completed survey received a $\$ 5$ gift card.

An investigator (Makowsky), blinded to SA-MRAQ responses, used a standardized data collection form to extract data from a printout of relevant sections of the clinic EMR (MedAccess, Kelowna, BC, Canada) produced within 1 week of survey completion and completed the EMR-MRAQ scoring. A second chart review was conducted to determine ER, hospital, and clinic visits 6 months after survey completion. This project was approved by the Health Research Ethics Board of the University of Alberta.

\section{Outcome Assessment}

Health Behavior/Status. Each of the validated measures were scored consistently with their original protocols. ${ }^{26-28}$ For the MMAS-8, a score of 3-8 was taken to indicate low adherence. ${ }^{26,29}$ "Inadequate health literacy" was defined as answering "often" or "always" to the question "How often do you have someone help you read hospital materials," which is effective in detecting inadequate health literacy as measured using the Short Test of Functional Health Literacy in Adults (area under the receiver operator curve $=0.87) .{ }^{27}$ Health distress and overall health scores ranged from 0-5, where higher scores indicate more distress and excellent health. ${ }^{28,30}$

Health Care Utilization. Two investigators (Makowsky and Wong) reviewed the "consults" section of the patient chart to determine the number of all-cause emergency or hospital visits and the "visits" section to determine the number of clinic visits. Hospital visits for routine procedures, such as colonoscopy were counted as consults not hospital admissions. The total number of clinic visits was calculated by summing the number of records, including those to see the physician, a nurse, or other health care team member.

\section{Statistical Analysis}

All versions of the MRAQ were scored dichotomously according to criteria outlined in Table 1 . Patents at high risk were defined as those with $\geq 3$ out of 5 possible "yes" responses on the 5-item questionnaires and $\geq 6$ out of 10 "yes" responses on the 10-item questionnaire. These thresholds reflect a majority of "yes" responses, and the 3 out of 5 threshold is consistent with other studies. ${ }^{20,23}$ MRAQ scores were not calculated when missing data were present. 
TABLE 1 Items Included in Self-Administered and Electronic Medical Record Medication Risk Assessment Questionnaires

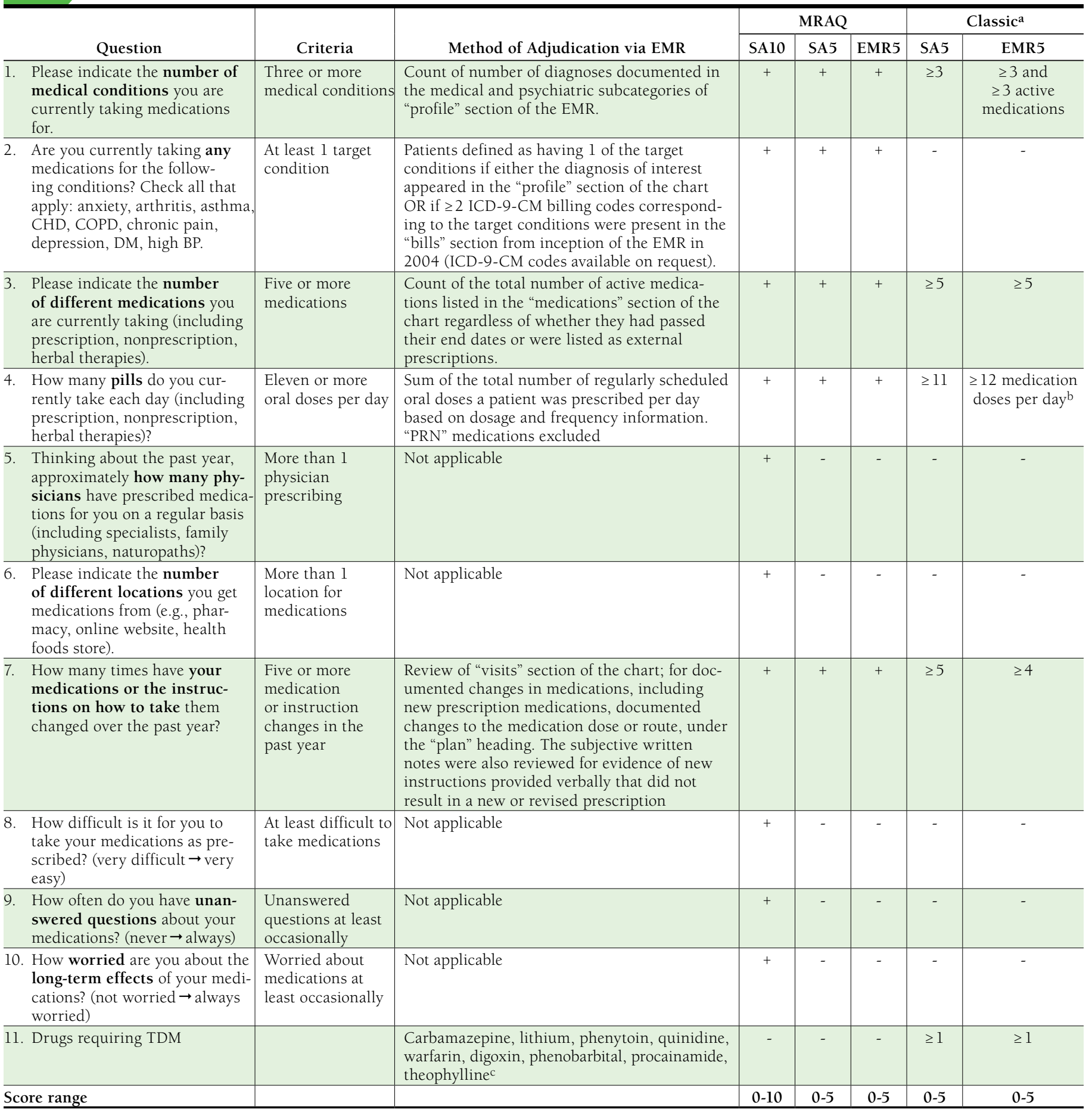

a Sensitivity analysis.

${ }^{b}$ Total number of doses for all regularly scheduled medications regardless of dosage form or route.

'This item was only gathered from the EMR and was not patient self-reported, so, the SA 5-item classic risk assessment is only an estimation.

$B P=$ blood pressure; $C H D=$ coronary heart disease; $C O P D=$ chronic obstructive pulmonary disease; DM=diabetes mellitus; EMR = electronic medical record;

ICD-9-CM = International Classification of Diseases, Ninth Revision, Clinical Modification; MRAQ=medical record medication risk assessment questionnaire;

$P R N=$ as needed; $S A=$ self-administered; TDM= therapeutic drug monitoring. 
TABLE 2 Test Characteristics for 5-Item EMR-MRAQ and 10-Item SA-MRAQ in Detecting Patients Characterized as High Risk on 5-Item SA-MRAQ

\begin{tabular}{|c|c|c|c|c|c|}
\hline \multirow[b]{2}{*}{ Criteria } & \multirow[b]{2}{*}{ Number } & \multicolumn{2}{|c|}{ Percentage of Charts Meeting Criteria } & \multirow[b]{2}{*}{ Sensitivity } & \multirow[b]{2}{*}{ Specificity } \\
\hline & & $\begin{array}{c}\text { Electronic Medical } \\
\text { Record } \\
\mathrm{n}(\%)\end{array}$ & $\begin{array}{c}\text { Self-Administered } \\
\mathrm{n}(\%)\end{array}$ & & \\
\hline \multicolumn{6}{|l|}{ 5-item MRAQ } \\
\hline$\geq 3$ medical conditions & 110 & $91 \quad(82.7)$ & $64(58.2)$ & 87.5 & 23.9 \\
\hline$\geq 1$ target condition & 140 & $136 \quad(97.1)$ & $131 \quad(93.6)$ & 99.2 & 33.3 \\
\hline$\geq 5$ medications & 129 & $88(68.2)$ & $78 \quad(60.5)$ & 80.8 & 51.0 \\
\hline$\geq 11$ pills per day & 140 & $48 \quad(34.3)$ & $26(18.6)$ & 84.6 & 77.2 \\
\hline$\geq 5$ medication changes & 140 & $46 \quad(32.9)$ & $9 \quad(6.4)$ & 77.8 & 70.2 \\
\hline Overall MRAQ $\geq 3$ criteria met & 104 & $70 \quad(67.3)$ & $55 \quad(52.9)$ & 81.8 & 49.0 \\
\hline \multicolumn{6}{|l|}{ Additional SA-MRAQ items } \\
\hline$\geq 1$ physician prescribing & 140 & - & $66 \quad(47.1)$ & - & - \\
\hline$\geq 1$ location & 140 & - & $29 \quad(20.7)$ & - & - \\
\hline$\geq$ difficult to take medications & 140 & - & $5 \quad(3.6)$ & - & - \\
\hline Unanswered questions $\geq$ occasionally & 140 & - & $29(20.7)$ & - & - \\
\hline Worried about medications $\geq$ occasionally & 141 & - & $61 \quad(43.3)$ & - & - \\
\hline Overall 10-item SA-MRAQ $\geq 6$ criteria met & 102 & - & $18 \quad(17.6)$ & 31.5 & 97.9 \\
\hline \multicolumn{6}{|l|}{ Sensitivity analysis } \\
\hline Overall classic $\geq 3$ criteria met & & $46 / 105 \quad(43.8)$ & $19 / 105 \quad(18.1)$ & 84.2 & 65.1 \\
\hline
\end{tabular}

Test statistics were calculated for the 5-item EMR-MRAQ and the 10-item SA-MRAQ in detecting patients characterized at high risk on the 5-item SA-MRAQ and for each individual criterion. The 5-item SA-MRAQ was considered the gold standard consistent with other reports. ${ }^{19,20,23}$ Correlation analysis was used to explore the strength of relationships between MRAQ scores and measures of health behavior/status. Finally, to determine how well MRAQ scores predicted patientimportant outcomes, including the composite of ER visits and hospitalizations, as well as a high number of future primary care clinic visits (i.e., $\geq 4$ visits in 6 months), low medication adherence, and inadequate health literacy, area under the receiver operating characteristic curve, sensitivity, specificity, and positive and negative predictive values were calculated for each outcome. Data were analyzed using SPSS version 23 for Mac (IBM Corp., Somers, NY).

\section{Post Hoc Sensitivity Analysis: Comparison of EMR-Classic and SA-Classic Risk Questionnaires}

To further explore the performance of the EMR-MRAQ and the overall proportions categorized at risk, we conducted a sensitivity analysis where we approximated the proportion of participants that would be categorized at high risk based on 5 "classic" criteria significantly correlated with the risk of medication-related problems and used by others (Table 1). ${ }^{19,20}$

\section{Results}

$\overline{\text { An estimated } 756 \text { patients attended the clinic while the survey }}$ team was present. Of these, 321 potential participants were approached, and 143 were included in the analysis, with a median age of 61 years (interquartile range $[\mathrm{IQR}]=51-72$ ) and $44 \%$ male, while 125 were ineligible, and 53 declined participation. Overall, 32.6\% $(n=47 / 138)$ had low medication adherence; $7.9 \%(n=11 / 140)$ screened positive for inadequate health literacy; and 34.3\% ( $n=48 / 140)$ rated their health as "poor" or "fair," with the median level of health distress as "low" (1.71 [IQR=0.75-2.50] out of 5), while 94\% were "sure" or "very sure" of their MRAQ responses. With respect to 6-month outcomes, $16.1 \%(n=23 / 143)$ had $\geq 1$ ER visit/hospitalization, while $37.5 \%$ ( $n=54 / 143)$ had $\geq 4$ clinic visits.

\section{Comparison of EMR-MRAQ and 10-Item SA-MRAO with 5-Item SA-MRAO}

In total, 104 (32\% of potential participants approached) completed all questions required to calculate both EMR- and SA-MRAQ scores. The EMR- and SA-MRAQs categorized 67.3\% and $52.9 \%$ of participants as high risk, respectively (Table 2). The EMR-MRAQ was $81.8 \%$ sensitive and $49 \%$ specific for detecting patients classified as high risk on the SA-MRAQ, and the sensitivity for each of the 5 criteria ranged from $77.8 \%$ to $99.2 \%$, while the specificity ranged from $23.9 \%$ to $77.2 \%$. The 10-item SA-MRAQ categorized $17.6 \%(n=18 / 102)$ of participants at high risk and had a $31.5 \%$ sensitivity and $97.9 \%$ specificity for detecting patients classified as high risk.

\section{Correlations Between MRAQ Scores and Health Behavior/Status Scales}

Overall, the 10-item SA-MRAQ had the strongest correlations with the measures of health behavior/status (medication 
adherence [Spearmans' rank correlation coefficient $\left(\mathrm{r}_{\mathrm{s}}\right)=0.198$, $P=0.049]$, health distress $\left[r_{s}=0.348, P<0.001\right]$, and overall health $\left[r_{s}=-0.392, P<0.001\right]$ ), while the 5-item SA-MRAQ and the EMR-MRAQ displayed weaker correlations with health distress and overall health (data not shown).

\section{MRAQ Performance for Patient-Important Outcomes}

Only the EMR-MRAQ was able to discriminate between those participants who would go on to have $\geq 1$ ER visit/hospitalization (c-statistic $=0.69 ; 95 \%$ confidence interval $[\mathrm{CI}]=0.57-0.81$ ), and it also had the highest ability to discriminate between those participants who would go on to visit the clinic $\geq 4$ times in 6 months (c-statistic $=0.77 ; 95 \% \mathrm{CI}=0.69-0.85$ ). The EMRMRAQ had high sensitivities ( $82.6 \%$ and $88.9 \%$ ) but low specificities (33.3\% and $42.7 \%$ ) for these outcomes, respectively. Only the 10-item SA-MRAQ was able to detect low medication adherence (c-statistic $=0.61 ; 95 \% \mathrm{CI}=0.50-0.72$ ) or inadequate health literacy (c-statistic $=0.69 ; 95 \% \mathrm{CI}=0.54-0.85)$ with low sensitivity (25.2\% and 22.2\%) and high specificity (86.4\% and $82.6 \%)$, respectively.

\section{Sensitivity Analysis}

As shown in Table 2, the EMR-classic and SA-classic questionnaires categorized fewer participants than the MRAQ at high risk, 43.8\% ( $n=46 / 105)$ and $18.1 \%(n=19 / 105)$, respectively. The EMR-classic questionnaire was $84.2 \%$ sensitive and $65.1 \%$ specific in detecting high risk on the SA-classic questionnaire. Both classic tools showed a similar pattern of correlations with health behavior/status outcomes and predictive ability for clinically important outcomes as their 5-item MRAQ counterparts (data not shown).

\section{Discussion}

In this study, we found that the EMR-MRAQ had high sensitivity and low specificity in detecting patients classified as high risk on the 5-item SA-MRAQ, was weakly correlated with health distress and overall health, and had fair ability to detect $\geq 1$ ER visit/hospitalization in 6 months and high clinic utilization with high sensitivity but low specificity. The 10-item SA-MRAQ identified fewer at risk, suffered from low sensitivity, and was able to detect outcomes that the EMRMAQ could not; however, its performance in detecting low medication adherence and inadequate health literacy was poor. While the EMR- and SA-classic risk criteria resulted in fewer patients labeled at risk in the sensitivity analysis, we are confident in our findings regarding the performance of the EMR-MRAQ because the EMR-classic version displayed similar sensitivity and specificity in detecting high-risk status using the SA-classic criteria. Overall, these results suggest that EMRbased risk stratification has acceptable performance compared with patient self-reporting.
Although we are the first, to our knowledge, to use a primary care clinic EMR review to calculate a medication risk score and explore correlations and ability to predict health care utilization outcomes, several others have explored computerized medication risk screening. ${ }^{31-35}$ The best known example comes from the IMPROVE investigators who validated a computer-based program to identify patients at high risk for medication-related problems using pharmacy dispensing records in ambulatory clinic patients attending the Denver Veterans Affairs Medical Center. ${ }^{31}$ They found that automated computer-based risk screening was $65.7 \%$ sensitive and $88.2 \%$ specific in detecting patients at high risk of medication-related problems, compared with a manual medical record review. While the IMPROVE investigators used 6 criteria (including assessment of compliance) known to correlate with medication-related problems, chart review was the gold standard, and patient-reported risk was not assessed. Two recent publications have explored electronic patient prioritization tools for clinical pharmacist interventions in hospitalized inpatients. ${ }^{34,35}$ Falconer et al. (2014) found that electronic monitoring of 38 clinical flags in virtual real time increased process measures, including the number of patients receiving medication reconciliation and discharge services and also prevented medication errors. ${ }^{34}$ Urbina et al. (2014) reported fair ability to detect patients with medication-related problems on a cardiology ward. ${ }^{35}$

In the ambulatory setting, Pammett et al. (2015) showed that a self-administered medication risk assessment based on classic risk criteria was reliable for detecting patients in community pharmacies who had a large number of medication-related problems. ${ }^{23}$ While we did not directly measure medication-related problems, we found that the SA-classic risk assessment had weak relationships with medication adherence, health distress, health literacy, and overall health and was only able to detect high clinic use. Pammett et al. also reported high levels of agreement between a patient self-administered risk assessment and a researcher-completed risk assessment based on a comprehensive medication assessment. ${ }^{23}$ Not surprisingly, we found lower agreement between a patient self-reported assessment and a researcher-completed EMR assessment, with the EMR assessment generally overestimating risk. This lower level of agreement may relate to problems with the accuracy or completeness of information contained within the EMR, which has been documented by other studies. ${ }^{36-38}$ For example, Kaboli et al. (2004) showed that only $5.3 \%$ of patients had complete agreement between their computer medication list and what the patient reported taking, ${ }^{37}$ and Orrico (2008) documented that the most common discrepancy is the presence of a medication that a patient no longer takes. ${ }^{38}$

\section{Limitations}

This study has several important limitations that need to be considered. The first limitation is selection bias. Although we approached consecutive patients to participate, the study occurred in a single academic clinic over a short period of time, 
and a large proportion of the patients were ineligible or declined participation. This lack of participation negatively affects the applicability of the study's findings to the spectrum of adult patients seen in primary care clinics but, more importantly, illustrates the greater potential of clinicwide EMR-based screening.

The second limitation relates to the validity of our adapted SA-MRAQ, since this tool had not been formally validated, and this study lacked an outcome relating to medication risk. Adapting our SA-MRAQ led to difficulty with patients answering certain questions and missing data for almost 30\% of participants. Based on this problem, one could question the reliability of the SA-MRAQ assessments even though a majority indicated a high degree of confidence in their answers.

Unlike other researchers, we decided against identifying medication-related problems via face-to-face medication reviews. ${ }^{19,23}$ This is a major limitation, and our sensitivity analysis suggested that both of the 5-item MRAQ tools overestimated risk because almost all reported at least 1 target condition. Although the MRAQ still requires future validation, we attempted to offset this by exploring patient-important outcomes. While "medication-related" or "preventable" ER/hospital visits may have been more optimal, we feel justified with our choice of all-cause ER/hospital visits, since Canadian data suggest that $24 \%$ of hospitalizations are drug related. ${ }^{7}$ While no cutoff point exists for defining an excessive number of primary care clinic visits in 6 months, our clinic data suggested an average return rate of 4 visits per 12 months, which justified the use of $\geq 4$ visits in 6 months.

Information bias is a third limitation. Bias was potentially introduced because the chart review was done by a clinician and not directly by the EMR. Although we did not use the Provincial Electronic Health Record (Netcare) to capture medications prescribed by outside clinicians or confirm outcomes, missing ER visits/hospitalizations is unlikely, since they are typically promptly available in the EMR. Finally, the 10-item SA-MRAQ suffered from lack of an accepted cutoff to define high risk, and confirmation bias brought on by the concurrent administration of measures may have contributed to its apparent ability to predict behavioral indicators.

\section{Conclusions}

An EMR-based medication risk assessment strategy shows potential in identifying patients at high risk of medicationrelated problems by self-reporting, correlates with health distress and overall health, and is able to detect patients who will use ER/hospital services or be high users of clinic services with high sensitivity but reduced specificity. Given the numerous advantages of an EMR-based strategy in terms of automation and efficiency in screening large panels of primary care patients, further development and validation of an EMR-based predictive model for patient-important medication-related adverse events using large administrative datasets is warranted.

\section{Authors}

MARK J. MAKOWSKY, BSP, PharmD, and KEN COR, PhD, Faculty of Pharmacy and Pharmaceutical Sciences, University of Alberta, Edmonton, Alberta, Canada. TAT WONG, BScPharm, Grey Nuns Community Hospital, Edmonton, Alberta, Canada.

AUTHOR CORRESPONDENCE: Mark J. Makowsky, BSP, PharmD, Faculty of Pharmacy and Pharmaceutical Sciences, University of Alberta, 3-171 Edmonton Clinic Health Academy, 1140587 Ave., Edmonton, AB T6G 1C9, Canada. Tel.: 780.492.1735;

E-mail:makowsky@ualberta.ca.

\section{DISCLOSURES}

This work was funded by the Canadian Society of Hospital Pharmacists Research and Education Foundation, which had no role in the analysis or interpretation of data or the decision to submit the manuscript for publication. The authors have no conflict of interests, potential or otherwise, to report.

Makowsky had full access to all of the data in the study and takes responsibility for the integrity of the data and the accuracy of the data analysis. Study concept and design were contributed by Makowsky and Cor. Makowsky and Wong collected the data, and data interpretation was performed by Makowsky, Cor, and Wong. The manuscript was written by Makowsky and was critically reviewed for intellectual content by Makowsky, Cor, and Wong.

\section{ACKNOWLEDGMENTS}

The authors acknowledge the physicians and staff of the Royal Alexandra Family Medicine Centre (Drs. Peter Bell, Joanne Caulfield, Julia Chronopolous, Keith Huber, Reagen Kijewski, Doug Klein, Sudha Koppula, Andrea Milne Epp, and David Moores) for supporting this project; Terry Legaarden for assisting in the recruitment of participants; Leslie Bortolotto and Shannon Wooley for providing guidance in the electronic medical record review; Drs. Scot Simpson, Lisa Guirguis, Mike Kolber, Neil Drummond, Tyler Williamson, Denise Campbell-Scherer, and Lee Green, along with Stephanie Garies, for providing methodological advice; and the EPICORE Centre for conducting the data analysis.

\section{REFERENCES}

1. Winterstein AG, Sauer BC, Hepler CD, Poole C. Preventable drug-related hospital admissions. Ann Pharmacother. 2002;36(7-8):1238-48.

2. Thomsen LA, Winterstein AG, Sondergaard B, Haugbolle LS, Melander A. Systematic review of the incidence and characteristics of preventable adverse drug events in ambulatory care. Ann Pharmacother. 2007;41(9):1411-26.

3. Tache SV, Sonnichsen A, Ashcroft DM. Prevalence of adverse drug events in ambulatory care: a systematic review. Ann Pharmacother. 2011;45(7-8): 977-89.

4. Roughead EE, Gilbert AL, Primrose JG, Sansom LN. Drug-related hospital admissions: a review of Australian studies published 1988-1996. Med J Aust. 1998;168(8):405-08.

5. Zed PJ, Haughn C, Black KJ, et al. Medication-related emergency department visits and hospital admissions in pediatric patients: a qualitative systematic review. J Pediatr. 2013;163(2):477-83.

6. Zed PJ, Abu-Laban RB, Balen RM, et al. Incidence, severity and preventability of medication-related visits to the emergency department: a prospective study. CMAJ. 2008;178(12):1563-69.

7. Samoy LJ, Zed PJ, Wilbur K, Balen RM, Abu-Laban RB, Roberts M. Drugrelated hospitalizations in a tertiary care internal medicine service of a Canadian hospital: a prospective study. Pharmacotherapy. 2006;26(11):1578-86. 
8. Choe HM, Farris KB, Stevenson JG, et al. Patient-centered medical home: developing, expanding, and sustaining a role for pharmacists. Am J Health Syst Pharm. 2012;69(12):1063-71.

9. Jorgenson D, Dalton D, Farrell B, Tsuyuki RT, Dolovich L. Guidelines for pharmacists integrating into primary care teams. Can Pharm J (Ott). 2013;146(6):342-52.

10. Kassam R, Martin LG, Farris KB. Reliability of a modified medication appropriateness index in community pharmacies. Ann Pharmacother. 2003;37(1):40-46.

11. Fishman PA, Goodman MJ, Hornbrook MC, Meenan RT, Bachman DJ, O'Keeffe Rosetti MC. Risk adjustment using automated ambulatory pharmacy data: the RxRisk model. Med Care. 2003;41(1):84-99.

12. Paulino EI, Bouvy ML, Gastelurrutia MA, Guerreiro M, Buurma H; Group E-SRCPR. Drug related problems identified by European community pharmacists in patients discharged from hospital. Pharm World Sci. 2004;26(6):353-60.

13. George J, Phun YT, Bailey MJ, Kong DC, Stewart K. Development and validation of the medication regimen complexity index. Ann Pharmacother 2004;38(9):1369-76.

14. Gordon KJ, Smith FJ, Dhillon S. The development and validation of a screening tool for the identification of patients experiencing medicationrelated problems. International J Pharm Pract. 2005;13(3):187-93.

15. Blalock SJ, Patel RA. Drug therapy concerns questionnaire: initial development and refinement. J Am Pharm Assoc (2003). 2005;45(2):160-69.

16. Pit SW, Byles JE, Cockburn J. Prevalence of self-reported risk factors for medication misadventure among older people in general practice. J Eval Clin Pract. 2008;14(2):203-08.

17. Rovers J, Hagel H. Self-assessment tool for screening patients at risk for drug therapy problems. J Am Pharm Assoc (2003). 2012;52(5):646-52.

18. Koecheler JA, Abramowitz PW, Swim SE, Daniels CE. Indicators for the selection of ambulatory patients who warrant pharmacist monitoring. Am J Hosp Pharm. 1989;46(4):729-32.

19. Barenholtz Levy H. Self-administered medication-risk questionnaire in an elderly population. Ann Pharmacother. 2003;37(7-8):982-87.

20. Langford BJ, Jorgenson D, Kwan D, Papoushek C. Implementation of a selfadministered questionnaire to identify patients at risk for medication-related problems in a family health center. Pharmacotherapy. 2006;26(2):260-68.

21. Makowsky MJ, Cave AJ, Simpson SH. Feasibility of a self-administered survey to identify primary care patients at risk of medication-related problems. J Multidiscip Healthc. 2014;7:123-27.

22. Snyder ME, Pater KS, Frail CK, Hudmon KS, Doebbeling BN, Smith RB. Utility of a brief screening tool for medication-related problems. Res Social Adm Pharm. 2015;11(2):253-64.

23. Pammett RT, Blackburn D, Taylor J, et al. Evaluation of a community pharmacy-based screening questionnaire to identify patients at risk for drug therapy problems. Pharmacotherapy. 2015;35(9):881-86.
24. Larrick A. CY 2016 medication therapy management program guidance and submission instructions. April 7, 2015. Available at: https://www.cms. gov/Medicare/Prescription-Drug-Coverage/PrescriptionDrugCovContra/ Downloads/Memo-Contract-Year-2016-Medication-Therapy-ManagementMTM-Program-Submission-v-040715.pdf. Accessed March 10, 2017.

25. Alberta Blue Cross. Compensation for pharmacy services. The Pharmacy Benefact, number 346. June 2012. Available at: http://www.rxa.ca/ media/24075/346_PSF.pdf. Accessed March 10, 2017.

26. Morisky DE, Green LW, Levine DM. Concurrent and predictive validity of a self-reported measure of medication adherence. Med Care. 1986;24(1):67-74.

27. Chew LD, Bradley KA, Boyko EJ. Brief questions to identify patients with inadequate health literacy. Fam Med. 2004;36(8):588-94.

28. Lorig KR, Sobel DS, Stewart AL, et al. Evidence suggesting that a chronic disease self-management program can improve health status while reducing hospitalization: a randomized trial. Med Care. 1999;37(1):5-14.

29. Morisky DE, Ang A, Krousel-Wood M, Ward HJ. Predictive validity of a medication adherence measure in an outpatient setting. J Clin Hypertens (Greenwich). 2008;10(5):348-54.

30. Ware JE, Snow KK, Kosinski M, Gandek B. SF-36 Health Survey: Manual and Interpretation Guide. Boston: The Health Institue, New England Medical Center; 1993.

31. Isaksen SF, Jonassen J, Malone DC, Billups SJ, Carter BL, Sintek CD. Estimating risk factors for patients with potential drug-related problems using electronic pharmacy data. IMPROVE investigators. Ann Pharmacother 1999;33(4):406-12.

32. Hammersley VS, Morris CJ, Rodgers S, Cantrill JA, Avery AJ. Applying preventable drug-related morbidity indicators to the electronic patient record in UK primary care: methodological development. J Clin Pharm Ther. 2006;31(3):223-29.

33. Bindoff IK, Tenni PC, Peterson GM, Kang BH, Jackson SL. Development of an intelligent decision support system for medication review. J Clin Pharm Ther. 2007;32(1):81-88.

34. Falconer N, Nand S, Liow D, Jackson A, Seddon M. Development of an electronic patient prioritization tool for clinical pharmacist interventions. Am J Health Syst Pharm. 2014;71(4):311-20.

35. Urbina O, Ferrandez O, Grau S, et al. Design of a score to identify hospitalized patients at risk of drug-related problems. Pharmacoepidemiol Drug Saf. 2014;23(9):923-32.

36. Wagner MM, Hogan WR. The accuracy of medication data in an outpatient electronic medical record. J Am Med Inform Assoc. 1996;3(3):234-44.

37. Kaboli PJ, McClimon BJ, Hoth AB, Barnett MJ. Assessing the accuracy of computerized medication histories. Am J Manag Care. 2004;10(11 Pt 2):872-77.

38. Orrico KB. Sources and types of discrepancies between electronic medical records and actual outpatient medication use. J Manag Care Pharm. 2008;14(7):626-31. Available at: http://www.jmcp.org/doi/10.18553/ jmcp.2008.14.7.626. 